\section{Dos miradas para el diseño de la marca destino Tisaleo}

\section{Resumen}

El presente artículo, derivado del proyecto de investigación "Diagnóstico de necesidades formativas en materia de ecoturismo y recursos naturales en la provincia de Tungurahua", aborda el proceso de creación de marca destino para Tisaleo, un pequeño cantón de la sierra andina ecuatoriana. El trabajo contrasta dos perspectivas sobre la imagen turística y su efecto como reclamo desde un enfoque cualitativo. Por un lado, un grupo de diseñadores gráficos locales proponen una serie de manuales de identidad corporativa que atienden a las características que han percibido como significativas en el cantón. Por otro lado, un equipo de investigación interdisciplinar e internacional elabora un informe sobre los atractivos potenciales para un segmento de viajeros interesados en el turismo basado en el "paisaje cultural". La disertación final concluye que la imagen de un destino turístico, además de representar las características propias del lugar físico, debe tener en cuenta que la mirada del visitante se pueda asociar en su imaginario a algún elemento evocador de lo que espera encontrar el perfil de viajero al que se quiere atraer.

* El presente trabajo forma parte del proyecto "Diagnóstico de necesidades formativas en materia de ecoturismo y recursos naturales en la provincia de Tungurahua", patrocinado por el Programa Prometeo de la Secretaría de Educación Superior, Ciencia, Tecnología e Innovación de la República del Ecuador.
Juan José Martí Noguera Doctor por la Universidad de Valencia. Investigador Prometeo, Universidad Técnica de Ambato. Ambato, Ecuador.

Correo electrónico:

juanjomn@gmail.com

\section{Daniel Díez Martínez}

Máster en Análisis, Teoría e Historia de la Arquitectura. Docente e Investigador, Facultad de Diseño, Arquitectura y Artes, Universidad Técnica de Ambato. Ambato, Ecuador. Miembro del grupo de investigación "Análisis y Documentación de Arquitectura, Diseño, Moda \& Sociedad", de la Universidad Politécnica de Madrid. Correo electrónico:

danieldiezmartinez@gmail.com

Christian Gabriel Ruiz Pérez Máster en Dirección de Comunicación Institucional y Empresarial. Docente, Facultad de Diseño, Arquitectura y Artes, Universidad Técnica de Ambato. Ambato, Ecuador. Correo electrónico: cg.ruiz@uta.edu.ec

\section{Verónica Elizabeth Chicaiza Redin \\ Doctora por la Universidad Complutense de Madrid. Facultad de Ciencias Humanas y de la Educación, Universidad Técnica de Ambato. Ambato, Ecuador. \\ Correo electrónico: \\ ve.chicaiza@uta.edu.ec}

Recibido: Agosto 2015

Aprobado: Noviembre 2015

Palabras clave:

Destino, imagen, marca, paisaje, turismo. 


\section{Two points of view for Tisaleo's brand design}

\begin{abstract}
This article, derived from the research project "Diagnosis of training needs for ecotourism and natural resources in the province of Tungurahua", addresses the creation process of Tisaleo, a small canton of Ecuador's Andean highlands destination brand. The study contrasts two perspectives on the touristic image and their effect as a claim from a qualitative approach. On one hand, a group of local graphic designers proposed a series of corporate identity manuals which address the characteristics they have perceived as significant in the canton. On the other hand, an interdisciplinary and international researchers team prepared a report on the potential attractive areas for a segment of travelers interested in tourism based on "cultural landscape". The final dissertation concludes that the image of a touristic destination, besides representing the characteristics proper of the physical location, should take into consideration that the visitors' gaze can associate in their imaginary some evocative element of what the visitor's profile who wants to be attracted expects to find.
\end{abstract}

Key words:

Destination, image, brand, landscape, tourism. 


\section{Introducción}

El presente trabajo presenta resultados parciales del proyecto de investigación "Diagnóstico de necesidades formativas en materia de ecoturismo y recursos naturales en la provincia de Tungurahua", financiado por el gobierno de Ecuador. Se aborda la dificultad de posicionamiento turístico de los municipios que integran la provincia, y mediante un trabajo organizado entre la Universidad Técnica de Ambato y autoridades locales se presenta el caso de creación y posicionamiento de la marca destino del municipio de Tisaleo. El proyecto de generar una imagen de marca destino pone en contraste la mirada de diseñadores gráficos autóctonos, en contraste con un grupo de investigación interdisciplinar que incorpora investigadores de diferentes regiones y países.

Tisaleo es un pequeño municipio de la provincia de Tungurahua, ubicada en la zona centro de la región interandina de la República del Ecuador, comúnmente conocida como sierra, unos cien kilómetros al sur de la capital, Quito. La provincia adquiere su nombre del volcán homónimo, un estratovolcán activo de 5.023 metros de altura que se integra dentro del Parque Nacional Sangay. Además de la "Mama Tungurahua", tal como se le conoce de acuerdo a la mitología indígena, la provincia cuenta con el nevado Carihuairazo, un volcán apagado de 5.020 metros de altura, así como con otros picos notables, entre los que destacan el Cerro Hermoso y el Igualata, de 4.480 y 4.432 metros de altura respectivamente.

Tisaleo es un municipio pequeño y poco poblado, que cuenta con 12.137 habitantes, de los cuales el 90\% vive en el área rural. El paisaje del cantón evidencia esta relación de entendimiento entre un modelo socioeconómico basado en la producción agrícola y ganadera minifundista y una naturaleza abrupta y de alto sentido escenográfico (Figura 1). 


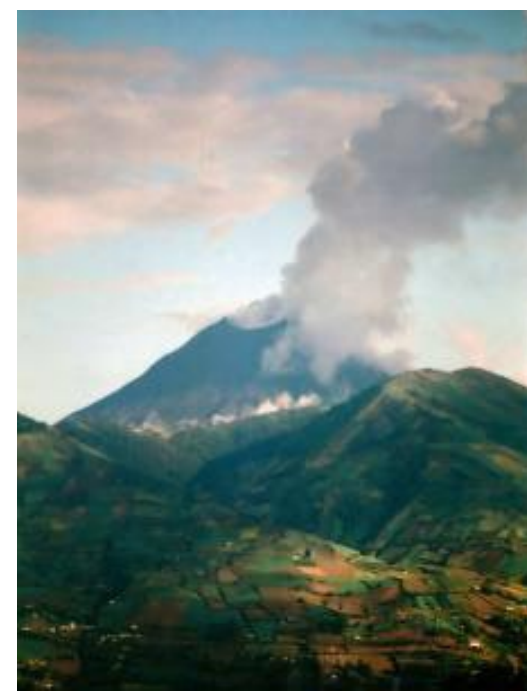

Figura 1. Vista del volcán Tungurahua desde Tisaleo. Fuente: archivo fotográfico de Víctor Hugo Navas.

Este vínculo con lo rural ha configurado un escenario social caracterizado por un elevado índice de pobreza que ha frenado el desarrollo del cantón, lo que igualmente ha contribuido a la preservación de su legado histórico patrimonial. Tisaleo ofrece una diversidad cultural que se singulariza en una fusión entre la cultura panzalea con la española, tal como ilustra la celebración de multitud de eventos y festividades. Ejemplo de ello es la conmemoración de las Octavas del Corpus Christi, en la cual se caracterizan diablos, indígenas y representantes de la cultura de raíces católicas (Figura 2), o la Fiesta del Inga Palla, declarada desde 2011 como Patrimonio Cultural Inmaterial del Ecuador, en la cual se escenifica el combate acontecido entre el cacique Tisaleo y los invasores españoles (Navas, 2000). Se trata, en ambos casos, de manifestaciones con una carga simbólica y ritual muy potente que preservan la convivencia de dos mundos coexistentes. 


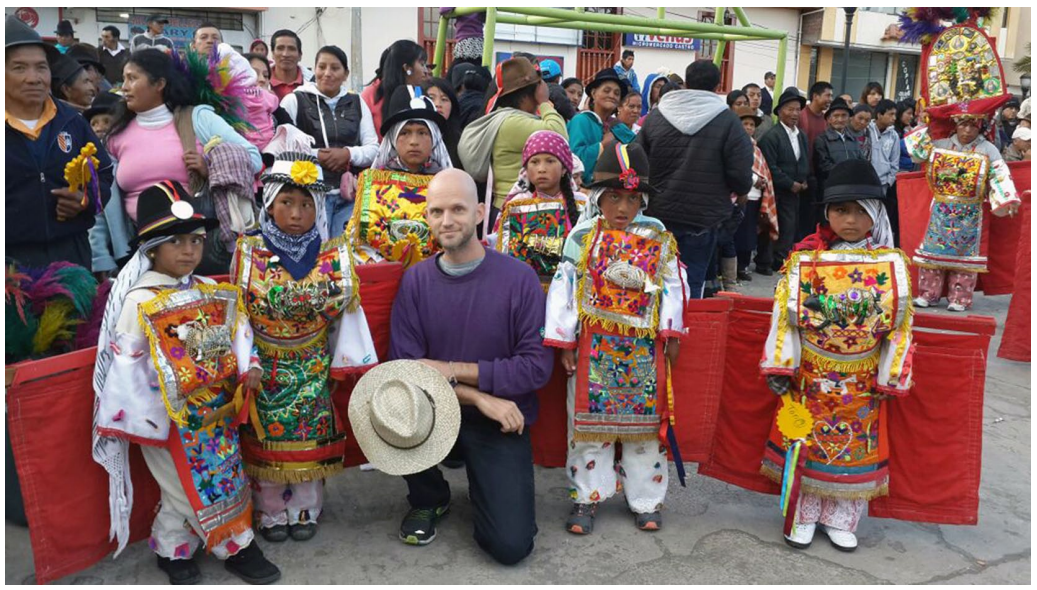

Figura 2. Turista extranjero durante las fiestas de las Octavas del Corpus Christi, 11 de junio de 2015. Fotografía: Rafael Sánchez.

El Plan Nacional del Buen Vivir o "Sumak Kawsay" en idioma quechua, desarrollado por la Secretaría Nacional de Planificación y Desarrollo de la República del Ecuador (Acosta, 2010) presta especial atención al respeto de la diversidad cultural del país y la convivencia armónica con el medioambiente. Se trata de un programa de desarrollo socioeconómico para el país que contempla, entre otras muchas estrategias, el impulso del ecoturismo basado en el rico legado natural, sociocultural y patrimonial del Ecuador (SENPLADES, s.f.).

En este escenario, el desarrollo de una oferta turística en Tisaleo se viene trabajando desde 2008 y desde entonces se ha orientado a visitas a fincas agrícolas y ganaderas, productoras de lácteos y artesanos. La articulación del producto turístico hacia activos como la riqueza cultural, la naturaleza o la fauna del cantón es aún incipiente, salvo en la anteriormente mencionada Fiesta del Inga Palla, que 
sí constituye un guiño al legado patrimonial y cultural que impregna el cantón. Además de los múltiples eventos y representaciones que se celebran a lo largo del año, Tisaleo cuenta con una arquitectura vernácula construida con técnicas prehispánicas de construcción con tierra que se ha preservado en el tiempo, y que desde 2009 se encuentra protegida por el Instituto Nacional de Patrimonio Cultural del Ecuador (INPC).

\section{La imagen del destino como catalizador del producto al turista}

El ecoturismo se define como la actividad caracterizada por el interés de visitantes a un destino motivados por la naturaleza y la cultura (Organización Mundial del Turismo \& Programa de las Naciones Unidas para el Medio Ambiente, 2002). La intervención del Estado ecuatoriano en el reconocimiento de las culturas que lo conforman y la preservación del medioambiente permiten abordar el proceso de cambio de matriz productiva desde un modelo económico extractivo con sobreexplotación de recursos naturales, hacia un proceso que permita poner en valor los atractivos intrínsecos a la articulación del mestizaje con el medio natural en torno al concepto de 'paisaje cultural' (Gómez, 2015), que en el caso concreto de Tisaleo, le confiere un poderoso atractivo para un segmento de viajero.

En este sentido, el diseño de una correcta estrategia de posicionamiento de la marca destino juega un papel fundamental, ya que tiene como misión atraer la mirada del turista hacia un lugar e influir en su decisión de viajar y conocerlo. Díaz (2003) considera que el consumo turístico se lleva a cabo a través de la mirada del viajero, que está organizada y sistematizada dependiendo de su procedencia, cultura y clase social. La mirada del turista sobre la imagen promocional pone su acento en las diferencias o similitudes con lo que conoce, en sus expectativas y en la interpretación de lo que espera encontrar para decidir viajar a ese lugar. Así, Guzmán, Garduño \& Zizumbo (2009) señalan que diseñar productos turísticos 
obliga no solo a identificar y promocionar los atractivos ecoturísticos y de recursos naturales, sino también a monitorear y redefinir el producto teniendo en cuenta al turista.

El proceso de creación de la imagen turística de un destino parte de un referente real en el que confluyen el espacio físico y las características medioambientales, económicas y socioculturales que lo configuran. De esa realidad nace la recreación de un imaginario en el cual se asientan las bases del producto turístico y se lleva a cabo una idealización de aspectos que se consideran atractivos y se enmascaran los aspectos menos agradables (Carballo, Moreno, León \& Ritchie, 2015). La imagen del destino debe acercar el ideal de un lugar para atraer al visitante hacia una experiencia por medio de emociones, a la vez que tener presente e integrar la percepción del turista y convertirla en un factor de atracción.

La dimensión estratégica de la imagen de destino conlleva considerar su efecto en el visitante en tres fases: a priori, antes de visitar; in situ, en el momento que contrasta expectativa y realidad encontrada; y a posteriori, cuando revive y decide compartir con otros sus fotografías, objetos adquiridos o anécdotas (Di Marino, 2008). Así pues, la imagen de destino puede tener una importancia crítica en el éxito o fracaso de la gestión de un lugar, haciendo necesario generar estrategias de investigación apoyadas en metodologías que atiendan múltiples variables para posicionar sus principales características como destino y orientarse al perfil de viajero (Ferreira, 2011).

\section{Metodología}

Este trabajo atiende a la creación imagen de destino de Tisaleo desde dos perspectivas con un enfoque cualitativo de observación y análisis. Por una parte, está la visión de los habitantes del lugar, que en este caso están representados 
por estudiantes de la carrera universitaria de Diseño Gráfico Publicitario de la Facultad de Diseño, Arquitectura y Artes de la Universidad Técnica de Ambato, en Tungurahua. Por otra parte, está el trabajo de un grupo de investigación interdisciplinar integrada por expertos en materia de arquitectura y turismo nacionales e internacionales. En el caso de los internacionales, cabe resaltar que no existía un conocimiento previo del lugar ni una imagen asociada al mismo que pudiera influir en la decisión de conocerlo.

El proceso metodológico seguido para el presente trabajo se estructura en tres momentos:

1. Establecimiento y delimitación de objetivos.

2. Trabajo de campo y desarrollo de producto.

3. Presentación y discusión.

El primer momento es una presentación del proyecto "Diagnóstico de necesidades formativas en materia de ecoturismo y recursos naturales en la provincia de Tungurahua", por parte del grupo de investigación interdisciplinar ante los estudiantes. Se sitúa en contexto el modelo de turismo basado en el concepto del "paisaje cultural", entendido como una combinación de atributos paisajísticos, arquitectónicos y sociales. El objetivo que se establece es la creación de una marca destino de Tisaleo.

En el segundo momento se lleva a cabo el trabajo de campo, que tuvo lugar de manera paralela por parte del grupo de investigación interdisciplinar y los estudiantes de Diseño Gráfico Publicitario. En ambos casos, se desarrolló el estudio sobre el terreno de las características del paisaje, así como entrevistas a actores del sector público y privado para recabar información sobre Tisaleo. Dicha labor se realizó de forma independiente y con la condición de resaltar los aspectos culturales y paisajísticos propios del destino. Además, los estudiantes trabajaron organizados 
en cuatro equipos independientes en la elaboración de un Manual de Identidad Corporativa, cuya estructura y contenido es la siguiente:

- Descripción procedimental acerca de los elementos básicos del identificador corporativo a desarrollar, donde se ofrezca una explicación técnica y fundamentada de los patrones de geometrización, simbología, texturas, colores y tipografías que emplean en el identificador.

- Normas básicas y restricciones para el uso correcto y unificado de los elementos gráficos que conformen la imagen de la marca diseñada, garantizando su independencia visual, así como una identificación rápida e inequívoca. Los estudiantes debían sustentar estas directrices atendiendo a criterios visuales y compositivos (tamaños mínimos, ejercicios de positivo/negativo y escalas de grises, manejo sobre colores planos, gamas cromáticas, etc.), y deben ejemplificarlo mediante la aplicación de la marca en material de oficina y papelería corporativa (carpetas, sobres, hojas membretadas, tarjetas de presentación).

- Maniobras de posicionamiento y promoción de la marca, como la realización de material P.O.P. y suvenires (bolígrafos, pins, cuadernos, tazas, camisetas, llaveros), la adquisición de visibilidad en redes sociales o las estrategias de participación en ferias de turismo a niveles provincial y nacional.

Finalmente, el tercer momento consistió en la presentación de los estudiantes de sus propuestas de identificador de la marca destino y el contraste de las miradas sobre la imagen captada por estudiantes versus los condicionantes del destino que resultan con poder de atracción para el grupo de investigación interdisciplinar. 


\section{Exposición de resultados}

A continuación, se exponen las dos miradas sobre Tisaleo.

\section{Mirada autóctona - estudiantes}

Se presenta, en primer lugar, el trabajo realizado por los cuatro grupos de estudiantes de Diseño Gráfico Publicitario de la Universidad Técnica de Ambato.

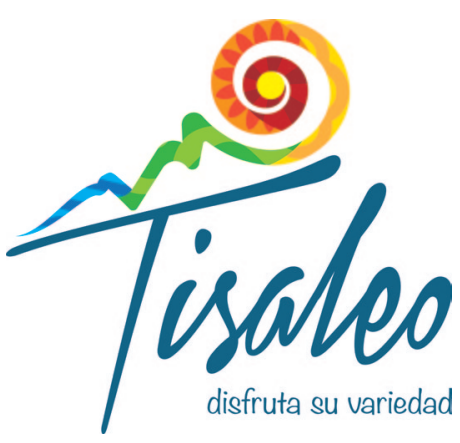

Figura 3. Imagotipo corporativo Grupo 1.

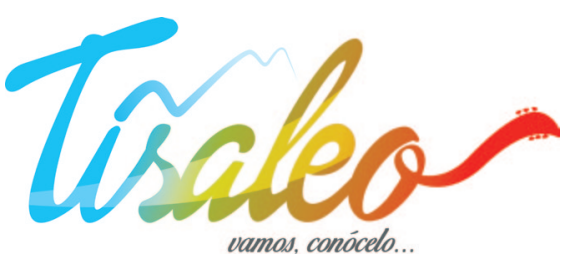

Figura 5. Imagotipo corporativo Grupo 3.

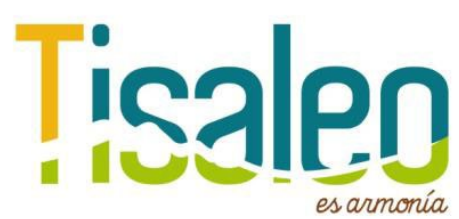

Figura 4. Imagotipo corporativo Grupo 2.

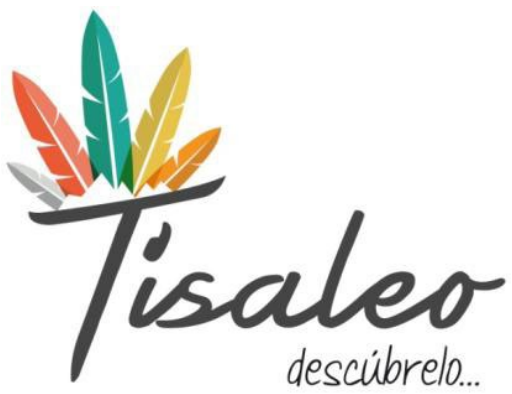

Figura 6. Imagotipo corporativo Grupo 4. 
El paisaje propio de Tisaleo ha sido un factor determinante para los estudiantes. Así, las cuatro propuestas de imagotipo integran una síntesis del característico perfil descabezado del volcán Carihuairazo', situado a solo 6,5 kilómetros de Tisaleo, desde donde es perfectamente visible en días claros y despejados. En el caso del isotipo del Equipo 1 (Figura 3), la línea que define el perfil del Carihuairazo se dibuja utilizando como base la parte superior de la T de Tisaleo. A continuación, esta línea se encierra sobre sí misma, a modo de espiral, como símbolo de unión y permanencia en el tiempo del cantón, a la vez que, mediante la utilización de una gama de colores cálida, juega con el concepto de la representación del sol en el mismo paisaje natural. Este isotipo presenta una variación de colores y texturas que se han inspirado en elementos propios de la naturaleza y la cultura del cantón: los lagos y nevados se representan con el color azul, los pastos y cultivos con el verde, la arquitectura patrimonial con el amarillo, el arraigo de la tradición con el naranja y la producción de objetos de artesanía con el rojo (Figura 7). Esas mismas texturas se utilizan, además de en el isotipo, en otras piezas gráficas y material P.O.P. (Figura 8).
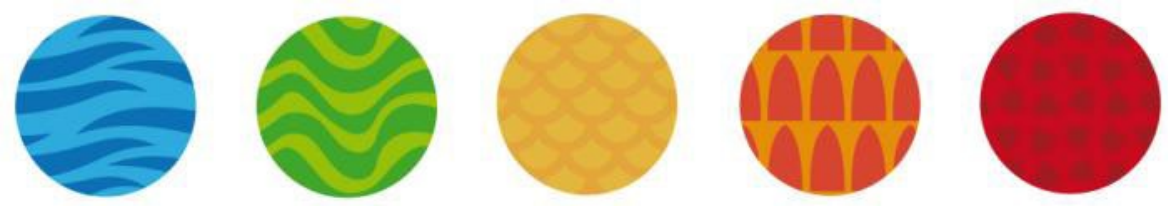

Figura 7. Colores y texturas presentes en el isotipo del identificador del Equipo 1.

\footnotetext{
${ }^{1}$ El Carihuairazo es un volcán apagado con nevados permanentes que tiene una altura de 5.116 metros. De acuerdo a la mitología indígena, que personifica los volcanes y los identifica con deidades sobrenaturales, el Carihuairazo presenta su cráter descabezado después de la pelea que mantuvo con el 'taita' Chimborazo por conseguir el amor de la 'mama' Tungurahua (Reino, 2000).
} 


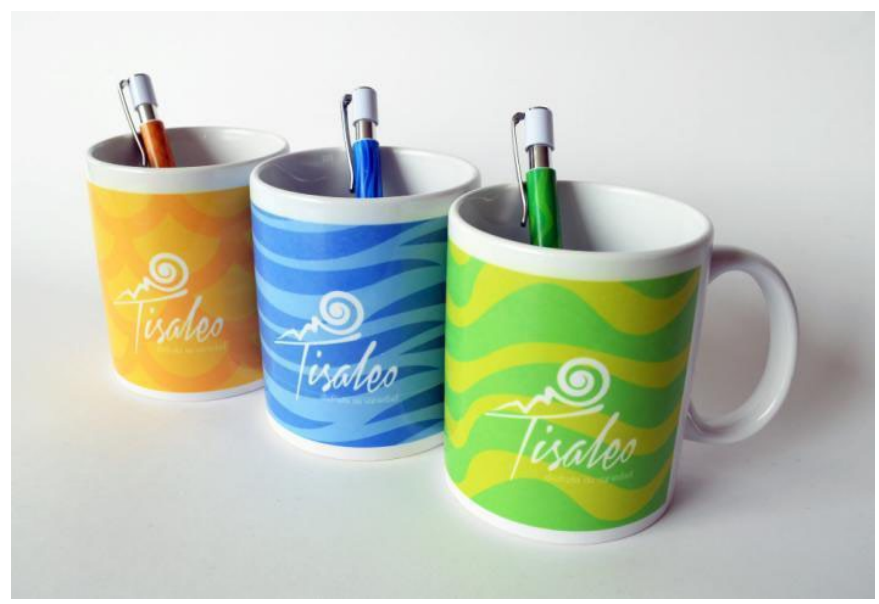

Figura 8. Aplicación en tazas de desayuno y bolígrafos de los colores y texturas presentes en el isotipo del identificador del Equipo 1. En estos ejemplos, el imagotipo aparece en blanco.

En la propuesta del Equipo 2 (Figura 4), el perfil del volcán se utiliza en color blanco, como un ejercicio de recorte que parte el isologo en dos y permite jugar con los colores y atributos típicos del paisaje, dejando así la parte inferior en verde (propio de los campos) y la superior en azul, como el cielo. La parte superior de la letra T aparece en amarillo, como el sol. Resulta llamativo cómo ese efecto de sustracción o recorte del perfil del volcán puede invertirse para adquirir relieve en otras aplicaciones, como por ejemplo la versión del isotipo en negativo en las piezas grabadas con láser sobre madera (Figura 9). 


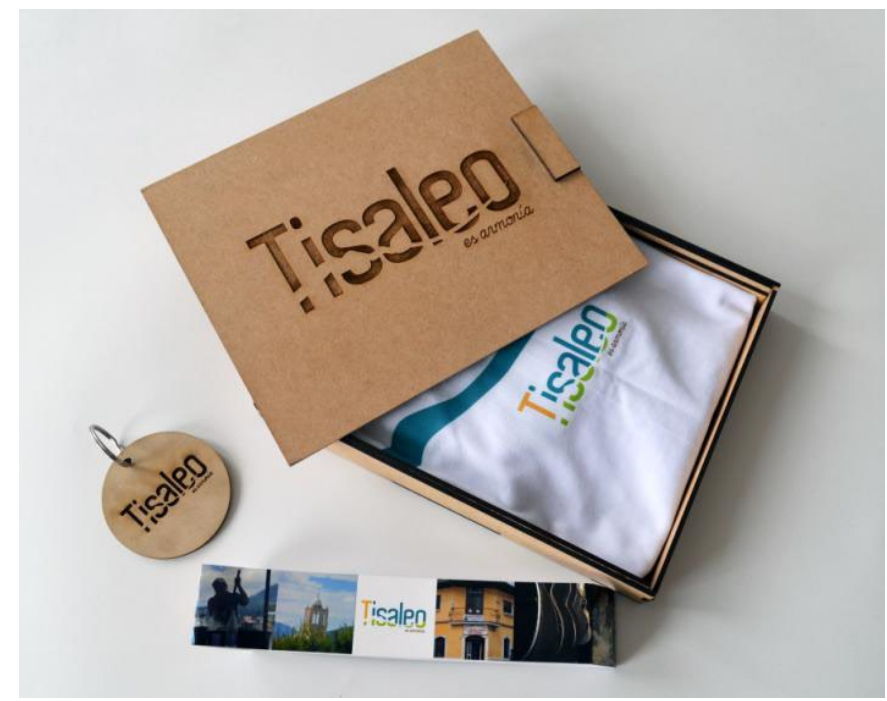

Figura 9. Kit de regalo consistente en camiseta y llavero incluidos en una caja de madera con la aplicación del identificador del Equipo 2. La línea del Carihuairazo adquiere relieve sobre las letras, grabadas con láser en la madera.

La propuesta del Equipo 3 (Figura 5) es un ejercicio de creación y modificación de una fuente tipográfica. El perfil del volcán es menos visible, y se presenta como un trazo sutil que surge de la letra "i" y se va desvaneciendo hacia la derecha, donde adquiere fuerza el mango de la guitarra, un producto artesano típico de Tisaleo, que se enlaza con el rabo de la letra O. La cromática utilizada se basó en los colores de la bandera de Tisaleo, en un ejercicio de degradado del color cian a verde y de verde a rojo (Figura 10). 

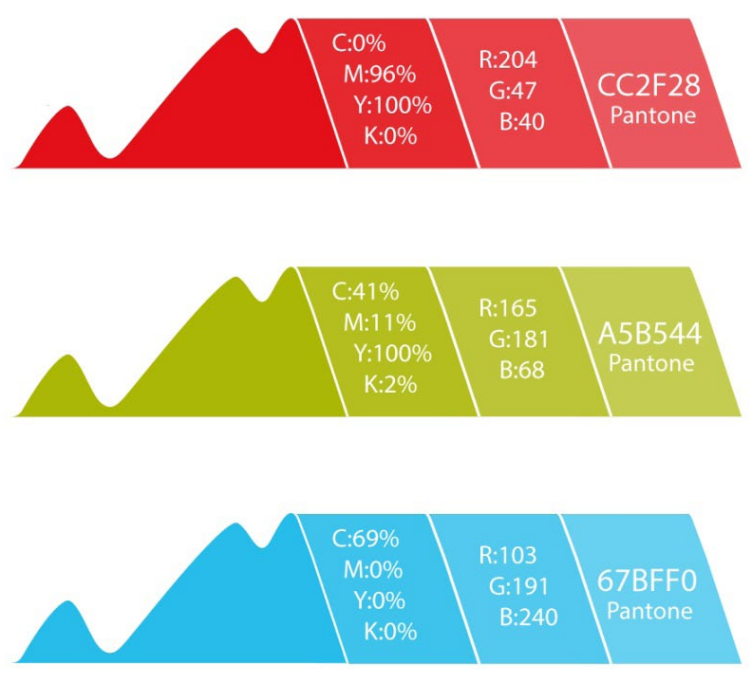

Figura 10. Cromática del identificador del Equipo 3.

Finalmente, en el caso del Equipo 4 (Figura 6), los estudiantes se remitieron a la historia del cantón para la construcción de su isotipo, para lo cual tomaron como elemento identificativo las plumas de la corona del cacique Tisaleo, cuya batalla contra los conquistadores españoles es el origen de la anteriormente mencionada Inga Palla, la fiesta más popular y representativa del cantón. La corona la componen cinco plumas de distinto color y es precisamente esta riqueza y capacidad de combinatoria cromática el rasgo más identificativo de la propuesta (Figura 11). El isotipo presenta en su parte inferior un recorte que, de nuevo, remite al perfil del Carihuairazo que, al igual que sucedía en la propuesta del Equipo 1, se apoya en la parte superior de la T de Tisaleo. 

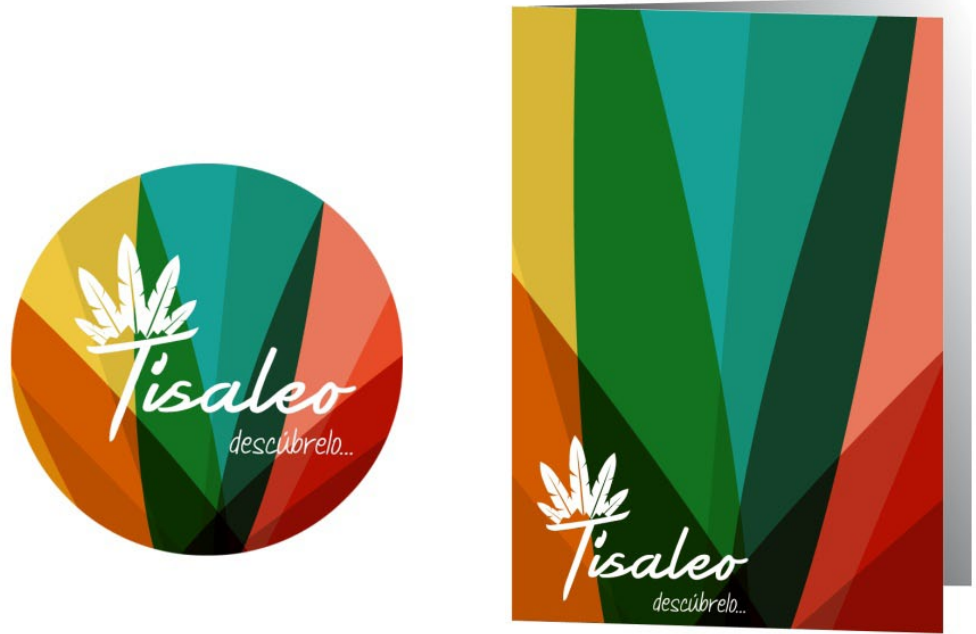

Figura 11. Aplicación del imagotipo en negativo blanco sobre combinación cromática en pins (izquierda) y en carpetas corporativas (derecha).

Con respecto a la tipografía primaria, los equipos 1, 3 y 4 utilizaron una caligráfica con serifa que permite establecer lazos con el carácter tradicional y artesano de Tisaleo. Por su parte, el Equipo 2 se distancia de este lenguaje y apuesta por un tipo de letra paloseco, que contrasta con la manuscrita empleada en el eslogan.

\section{Mirada del turista - grupo de investigación interdisciplinar}

El grupo de investigación interdisciplinar concretó su trabajo de campo en un informe que recoge las principales características de Tisaleo como paisaje cultural, y el perfil de turista atraído por lugares singulares que conformen un paisaje 
cultural, atendiendo a la Convención sobre la Protección del Patrimonio Mundial Cultural y Natural (UNESCO, 1972). El informe resalta, primeramente, que Tisaleo resulta un lugar desconocido por la escasa información previa que se puede hallar por búsquedas de información a través de la Red. A priori, el principal referente resulta en imaginarios de la cordillera andina y Ecuador, sin centrar la atención en un punto concreto. Por lo tanto, se accede a documentación turística por medio de materiales editados por el municipio y el comité provincial de turismo de Tungurahua. Finalmente, el trabajo de campo confronta las expectativas entre lo leído e imaginado, y la realidad que se va descubriendo en recorridos y entrevistas con actores del sector.

El informe de las visitas realizadas durante el estudio resalta principalmente dos aspectos señalados como factores de potencial interés turístico cultural: la diversidad multicultural indígena-mestiza y el paisaje conformado por la acción de la mano del hombre sobre la tierra por medio de una gama de colores y texturas producto de los diferentes cultivos (Figura 12). Se destaca para el ecoturismo la fuerza icónica del paisaje y su relación con la población que lo habita, y su nexo entre lo esperado en los Andes y lo hallado en Tisaleo.

Del mismo modo, la arquitectura vernácula andina (Figura 13) constituye un importante atractivo que alimenta un imaginario que remonta hacia un momento histórico de cruce de civilizaciones en el cual la civilización incaica inició un proceso de resistencia, convivencia y mestizaje con la migración europea (Zamudio, 2013). Esta información histórica constituye un atractivo para un perfil de turista cultural, por lo que darla a conocer puede reforzar el interés por Tisaleo al ser uno de los lugares en los cuales se desarrolló una de las últimas batallas de resistencia entre los incas y españoles. 


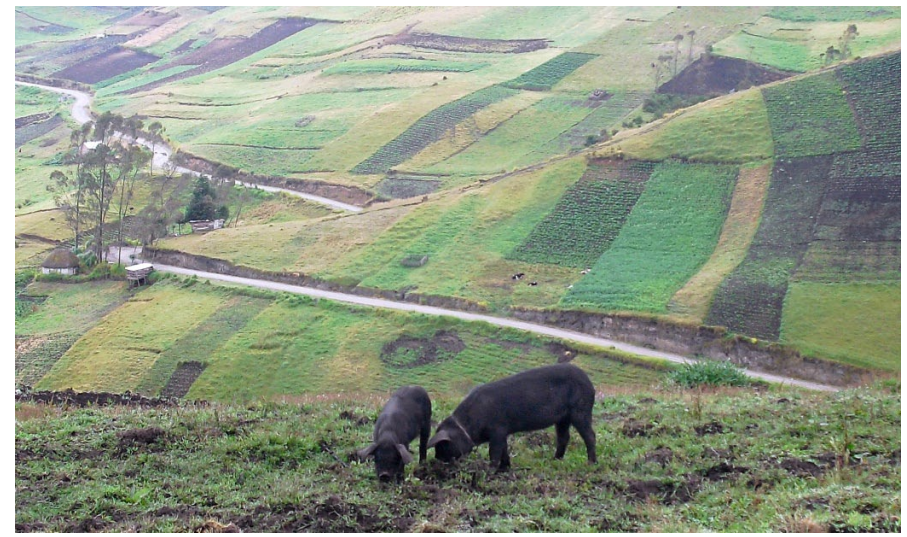

Figura 12. El valor del paisaje trabajado por la mano del hombre, campos de cultivo en Tisaleo. Fotografía: Juan José Martí.

Los argumentos presentados por el grupo de investigación resaltan la importancia de orientar el desarrollo del ecoturismo atendiendo a conocer el perfil de viajero, considerando prioritario que una marca destino incorpore algunos elementos que contribuyan a identificar algún rasgo con poder de imagen. Así como la imagen de "Juan Valdez" para el café colombiano, en el caso de la sierra andina no se ha encontrado información que permita que un municipio como Tisaleo sea ubicado turística o geográficamente. Ante ello, se considera necesario el tener presente en el diseño de marca dicha debilidad, ante la cual puede contrarrestar en positivo la imagen de cordillera andina y su paisaje cultural e histórico como el relatado caso de la resistencia inca. 


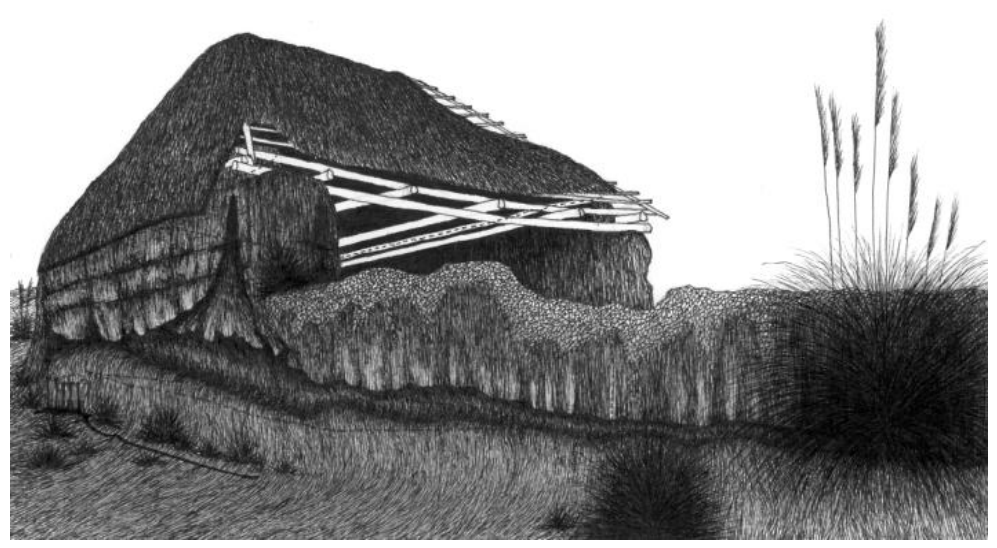

Figura 13. Ilustración de una vivienda tradicional andina, con estructura de muros de bahareque y cubierta de carrizo y paja. Ilustración: Jimmy Silva.

\section{Conclusiones}

La decisión de realizar un viaje y elegir entre varios destinos resulta un proceso complejo en el que interfieren múltiples variables y una extensa oferta. Como se ha comentado anteriormente, la imagen de destino puede resultar decisiva para incrementar el deseo de conocerlo. Ello implica reflexionar acerca de cómo la influencia de la imagen previa del destino que se transmite al turista es decisiva en la búsqueda de información (Frías, Rodríguez, Castañeda, Sabiote \& Buhalis, 2012).

La investigación que se plasma en este artículo permite contrastar el efecto del imaginario de la población local con la visión apriorística del posible visitante extranjero a través del proceso de creación de marca destino. Así, los estudiantes de Diseño Gráfico Publicitario orientan la imagen turística resaltando 
fundamentalmente el valor paisajístico de Tisaleo, con el volcán Carihuairazo como exponente más significativo. Sin embargo, el grupo de investigación interdisciplinar pone su atención turística en las gentes y su acción sobre el medio, y no solo en el referente geográfico del volcán.

Como se ha dicho anteriormente, la mirada del turista nunca es neutra. Al contrario, tiene algún imaginario o representación del lugar y expectativas sobre lo que espera encontrar (Urry \& Larsen, 2011). En los estudiantes, residentes en la provincia de Tungurahua, los volcanes tienen una carga simbólica importante, por lo que diseñan una imagen de marca destino desde el entendimiento de que el turista verá el paisaje desde su misma óptica. No obstante, para el grupo de investigación, que incluye internacionales para los cuales Ecuador no tiene una asociación o carga emocional previa con el volcán, el paisaje cultural tiene un referente icónico mucho más poderoso en informaciones previas genéricas, como la cordillera andina, o más específicas, como la cultura indígena ecuatoriana o el mundo prehispánico que incluye al imperio Inca.

El diseño de marca destino puede orientarse a un perfil genérico de visitante, que se contenta en atraer y mostrar los productos. Mientras que el objetivo del turismo cultural no trata únicamente del rendimiento económico que se pueda obtener al atraer turistas, sino que conecta al visitante extranjero con los valores idiosincráticos locales puede y debe constituir un impetuoso factor de conservación y recuperación de una tradición y patrimonio histórico que, a juicio de los integrantes del grupo de investigación interdisciplinar, tiene un valor incuestionable. Del mismo modo, la estrategia corporativa de la marca destino Tisaleo debe incluir estas reflexiones y convertirse en una herramienta de acercamiento a ese mundo, comprendiendo quiénes son los posibles receptores de una campaña de márquetin a los cuales debe provocar la imagen un interés en conocer el destino. 
Por lo tanto, y a modo de conclusión, a juicio de los autores de este artículo las estrategias corporativas propuestas por los estudiantes no alcanzan a relacionar un destino poco conocido con la imaginación del potencial turista extranjero a quien va dirigida la imagen. Si bien son ejercicios de diseño atractivos, visual y compositivamente potentes, todos proponen aproximaciones con significados excesivamente genéricos, que no reflexionan acerca de la importancia de que la estrategia de diseño tenga presente las expectativas del turista para orientar la mirada hacia un lugar único.

\section{Referencias}

Acosta, A. (2010). El Buen Vivir en el camino del post-desarrollo: Una lectura desde la Constitución de Montecristi. Policy Paper 9. Quito: Fundación Friedrich Ebert Stiftung - Instituto Latinoamericano de Investigaciones Sociales. Recuperado de http://library.fes.de/pdf-files/bueros/quito/07671.pdf

Ayora, S. I. (2003). Medicina, nostalgia y la mirada turística en Chiapas. En C. Bueno Castellanos \& E. Aguilar Criado (Coords.), Expresiones locales de la globalización: México y España (pp. 355-380). México, DF: Centro de Investigaciones y Estudios Superiores en Antropología Social - Universidad Iberoamericana.

Carballo, R., Moreno, S., León, C. J., \& Ritchie, B. (2015). La creación y promoción de experiencias en un destino turístico. Un análisis de la investigación y necesidades de actuación. Cuadernos de Turismo, 35, 71-94.

Di Marino, E. (2008). The Strategic Dimension of Destination Image. An Analysis of the French Riviera Image from the Italian Tourists Perceptions. (Tesis doctoral). Università degli Studi di Napoli "Federico II", Nápoles, Italia. 
Ferreira, S. D. (2011). Destination image: Origins, Developments and Implications. Revista de Turismo y Patrimonio Cultural, 9(2), 305-315.

Frías, D. M., Rodríguez, M. A., Castañeda, J. A., Sabiote, C. M., \& Buhalis, D. (2012). The Formation of a Tourist Destination's Image via Information Sources: the Moderating Effect of Culture. International Journal of Tourism Research, 14, 437-450.

Gómez, A. (2015). Modelo de diseño para la valoración y apropiación social del Patrimonio en el Paisaje Cultural Cafetero colombiano. Revista Kepes, 11, 117-138.

Guzmán, C., Garduño, M., \& Zizumbo, L. (2009). Reflexión crítica sobre el consumo turístico. Estudios y Perspectivas en Turismo, 18(6), 691-706. Recuperado de http:// www.redalyc.org/pdf/1807/180713900004.pdf

Navas, V. H. (2000). Tisaleo: cultura y tradición. Consejo de desarrollo de las nacionalidades y pueblos del Ecuador. Quito, Ecuador.

Organización Mundial del Turismo \& Programa de las Naciones Unidas para el Medio Ambiente. (2002). Cumbre Mundial del Ecoturismo: Informe Final. Madrid: Organización Mundial del Turismo. Recuperado de http://www.unep.fr/shared/ publications/pdf/WEBx0079xPA-EcotourismSummitES.pdf

Reino, P. A. (2002). Tisaleo indígena en la colonia. Ilustre Municipio del Cantón Tisaleo-Tungurahua, Ambato, Ecuador.

Secretaría Nacional de Planificación y Desarrollo (SENPLADES). (s.f.). Plan Nacional del Buen Vivir 2013-2017. Quito, Ecuador. Recuperado de http://documentos. senplades.gob.ec/Plan\%20Nacional\%20Buen\%20Vivir\%202013-2017.pdf

UNESCO. (1972). Convención sobre la Protección del Patrimonio Mundial Cultural y Natural. París. 
Revista KEPES, Año 12 No. 12, julio-diciembre de 2015, págs.331-352

Urry, J., \& Larsen, J. (2011). The Tourist Gaze 3.0. London: Sage Publications.

Zamudio, L. S. (2013) Arquitectura y turismo. La arquitectura como reclamo turístico. Urbano, 28, 58-67. Recuperado de http://www.redalyc.org/articulo. oa?id=19836965009

Cómo citar este artículo:

Martí, J. J., Díez, D., Ruiz, C. G. \& Chicaiza, V. E. (2015). Dos miradas para el diseño de la marca destino Tisaleo. Revista Kepes, 12, 331-352. DOI: 10.17151/kepes.2015.12.12.16 